\title{
THE ROLE OF METABOLISM IN AGING
}

\author{
Leo S. Luckinbill and Patricia Foley \\ Biological Sciences \\ Wayne State University \\ Detroit, MI 48202
}

\begin{abstract}
Metabolism appears to play a significant role in determining the rate of aging. Long-lived mutants and selected stocks of model experimental organisms exhibit characteristic changes in life history and physiological features consistent with altered metabolism. Specifically, these include the accumulation of metabolite pools of glycogen, lipid and polyhedric alcohols, suggesting that prolonged life is associated with restriction of the flow of carbon through glycolysis. Limiting carbon flow by reducing caloric intake is well known to extend life. Studies examining the mRNA expression pattern of functional gene groups generally indicate that nutrient restriction does affect metabolism. One study of Drosophila melanogaster has demonstrated that the flux of carbon through glycolysis is reduced in larvae of selected long-lived populations.

Here we propose a new hypothesis describing the interaction between the glycation process, glucose level, damage by free oxygen radicals and chaperonin proteins. Intermediate steps of the Maillard Reaction produce free radicals, similar to those produced during respiration, that also damage lipids, proteins and DNA. Antioxidant enzymes themselves can be inactivated by glycation. This establishes a positive feed-back between the rate of metabolism, glucose availability and damage by free oxygen radicals. The high levels of available glucose in ad libitum feeding should add to production of radicals, reduce levels of protective antioxidants and proportionally increase damage by free radicals. Thus, caloric restriction should lower available glucose, increase the level of antioxidants and thereby slow the rate of aging. Chaperonins act to block glycation, reducing its negative effect on antioxidant enzymes release and contribution of free radicals.
\end{abstract}

\section{INTRODUCTION}

The study of aging has progressed further and faster in the last decade than in the entire previous century. The application of molecular genetic methods to a number of

To whom all correspondence should be addressed:

Dr. Leo S. Luckinbill

Biological Sciences, Wayne State University

Detroit, MI

Tel.: (313) 577-2910

E-mail: lluckin@lifesci.wayne.edu elegant experimental models has disclosed the identity of several influential genes. We have also gained a limited knowledge of a larger set of participating genes and are forming a basic understanding of the link between genes that control the rate of aging and evolution. Despite these strides, however, modern research still lacks a single unrivaled concept of the mechanism of senescence.

The study of aging and senescence has always been replete with hypotheses about its basic mechanism. Early theories focused on physiological aspects of aging, but many were little more than circular restatements of the features observed in the aging process itself. Early examples of this can be found in the "waste elimination" (Metchnikof, 1908) and "essential nutrient depletion" (Molisch, 1938) hypotheses; that aging occurs respectively because of the improper (and incomplete) elimination of metabolic wastes or exhaustion of essential nutrients from tissues. The consequences of incomplete waste elimination are as much a result of the aging process and failing soma as they are a cause of other associated ill effects.

Such hypotheses fall far short by modern standards, but they point to a problem that is still evident. Senescence occurs as the net result of a cascading sequence of events that widens into various systems in its effect and accelerates with chronological age. Effects that issue from an underlying first cause, initiate other changes. Those in turn, create others that give senescence its expanding, self-propagating quality. This property of the aging process has caused difficulty in distinguishing clearly between cause and effect and has, therefore, hindered the design of experiments capable of resolving between alternative hypotheses.

Since aging and senescence are essentially deterioration, relatively few essentials are necessary for proposing a hypothesis of senescence. The proposed mechanism should explain the following observations: (1) Aging is spontaneous and nonsystematic in the particular aspects of deterioration that occur. That is, failure should be nonsystematic in kind or with respect to tissue, organ, system and age. Aging is a generalized phenomenon, not confined to particular tissues, organs or organ systems. Individuals, populations, species and higher taxa should exhibit variation in the rate of aging both within and between groups. (2) The effects of the proposed mechanism should increase with chronological age and at an accelerating rate. Irrespective of the mortality models that senescence can be described to fit, the mortality rate for most of the population and for 
most ages should increase at an accelerating pace. (3) The mechanism should cause deterioration that is largely irreversible. Different rates of aging only reflect the accumulation of damage at different rates. While specific effects of aging may be made to accumulate more slowly under certain environmental conditions, they too are irreversible. (4) A unified mechanism should explain or at least be consistent with the known empirical features of aging. For example, it should be consistent with the phenomenon of nutrient or caloric restriction as well as life-span extension induced by sublethal heat shock. (5) Proposed mechanisms should include the consideration of a genetic basis with intrinsic variability in which genes control and modify the rate of aging and respond to selection pressure in evolution. While all these features seem demanding in their collective specificity, these prescribed conditions appear to be met by at least two hypotheses.

(1) The first, and least investigated mechanism attributes senescence to the effects of problems caused by the use of glucose in metabolism (Cerami, 1985). While glucose is not ordinarily considered to be either a particularly unstable molecule nor especially detrimental in its action, it can exist in stable confirmations that are unquestionably destructive in their involvement in metabolism. In the process of nonenzymatic glycation, free glucose undergoes transformation in the Maillard Reaction, first to a Schiff's base, but eventually assuming stable conformations called Amadori arrangements and finally, the most stable Advanced Glycosylation Endproducts (AGEs). These products of glycation form crosslinkages rendering the proteins, lipids or DNA to which they are attached nonfunctional. The process occurs spontaneously, depends on the availability of free glucose in blood, tissues and the cytosol, is progressive, irreversible and cumulative in the damage it causes.

2) The second and most widely investigated mechanism comes from the damage cause by Reactive Oxygen Species (ROS) and so-called "free radicals" (Harman, 1956). Respiration and use of oxygen by aerobic organisms is not without definite cost. The partial reduction of molecular oxygen during oxidative phosphorylation in the mitochondria produces three major ROS; $\mathrm{O}_{2} \cdot \mathrm{H}_{2} \mathrm{O}_{2}$ and $\bullet \mathrm{OH}$. Of these, $\mathrm{H}_{2} \mathrm{O}_{2}$ and the radical derived from it, $\bullet \mathrm{OH}$, are believed to be the most destructive, particularly in their action on mitochondrial membranes. In addition to the damage they cause themselves, these three ROS also produce a group of other short-lived, highly oxidative radicals, that also inflict extensive damage on essential biological molecules. Damage to mitochondrial DNA has been estimated to occur at ten times the level of that for nuclear DNA (Shigenaga et al, 1994). Together these ROS and their derivatives can cause proteins to lose their sulfhydral $(-\mathrm{SH})$ groups or to be carbonylated. This can inactiviate them as enzymes or subject them to proteolysis (Stadtman, 1992). ROS can also peroxidate the polyunsaturated fatty acid chains of membranes, terminating their function (Sagai and Ichinose, 1980). This is thought to be the source of the brown lipofuscin pigments, widely considered to be an indicator of aging. DNA can be critically changed in several ways ranging from alteration of base pairs to strand breaks and crossovers (Ames et al, 1993; Aganwal et al, 1994). The oxidative damage to proteins, lipids and DNA are all detectable and have been shown to vary among individuals, species and phylogenetic groups and to increase with age. Damage inflicted by ROS is believed to be implicated as the cause of a number of degenerative diseases including Parkinson's disease, amyotrophic lateral sclerosis (Lou Gehrig's disease) and others (Yim et al, 1996; Wallace, 1996; Simonian and Coyle, 1996). Though the activity in antioxidant enzymes may not correspond to its predicted role in aging (Matsuo et al, 1992), the "free radical" hypothesis clearly has a reasonable basis in documentation of the disastrous effects of oxygen use (Jazwinski, 1996). Support for this hypothesis is not only provided by the the comparative patterns of damage that correlate with effects in aging or disease, but also by the effects of experimental manipulation of levels of free radicals in tissues..

\section{The Effects of Experimentally Varied ROS Levels in Model Systems}

Another category of evidence is found in experiments manipulating free radical levels and consequent oxidative damage to tissues. The mechanism of toxic action in eukaryotes of the herbicide paraquat is well understood. In a hydrated biological system, paraquat generates $\mathrm{H}_{2} \mathrm{O}_{2}$, as well as the radicals that issue from it. These are assumed to arise and act in ways that are identical to the $\mathrm{H}_{2} \mathrm{O}_{2}$ and radicals that are generated naturally in respiration, but at the greater levels imposed in experiments. When ingested or taken up by an organism, the effects on longevity are devastating and immediate. Paraquat reduces both the maximum and average life-span. Its effect is assumed to accelerate the oxidation undergone in natural aging.

Evidence that the ability to resist oxidative radicals enhances life-span comes from studies that apply tests to long-lived genetic variants or stocks. In this methodology, long-lived mutants or stocks are created in experimental model systems, by either mutagenesis or selection. The gene(s) of interest is identified by conventional genetic methods and considered for its relationship to known metabolic pathways and other Longevity Assurance Genes (LAGs), particularly those that give resistance to ROS. This is then followed with a series of environmental challenges to evaluate the ability of the long-lived individuals to withstand the saturation levels of ROS that come from paraquat. These tests presumptively assess the ability of a given genetic strain or stock to withstand the stress of what is believed to be an environmentally forced, accelerated pace of aging.

In the paraquat test, life-span is typically seen to decline in both long-lived experimental populations and in short-lived control variants. But, individuals that would have been long-lived under normal circumstances are 
usually resistant to it and though their life is shortened, they maintain a relatively greater maximum and average longevity than short-lived controls. Control populations fare even worse and die sooner than the experimental group. The test for resistance to the free radicals of paraquat has been applied to an impressive list of strains with genes and signal transducing elements suggesting that: (1) diverse genetic pathways are employed in achieving long life, (2) extended life-span usually also confers an enhanced defense against oxygen radicals, and (3) extended life-span varies widely across phylogenetic groups.

The process of aging is not viewed in all model systems from preceisely the same standpoint. In the lower eukaryotes, aging is considered from the standpoint of the clone, rather than as a senescing population of individuals. For example, aging is determined for Podospora anserina as the ability of mycelia to grow rapidly and continuously (Osiewacz, 1995). Aging cultures grow more slowly, turn brown and eventually reproduce sexually before death. In Saccharomyces cerevisiae, aging is determined by the number of times an individual cell divides. The number of cell divisions are indicated by the bud scars, which are formed as cell division occurs. As the number of cell divisions increases, growth slows and death finally occurs. In Neurospora crassa, the state of senescence is measured by the number of viable conidial spores (Jazwinski, 1990,1996).

While a number of genes in these lower eukaryotes do provide protection against free radicals, they seem to bear no outstanding relationship to longevity. For example the ATX1-2 genes of $\underline{S}$. cerevisiae give resistance to oxygen toxicity, but are unrelated to life-span, as defined (Lin and Culotta, 1995; 1996). Alternatively, a number of genes have been discovered affecting lifespan, but have no special relationship to oxygen radical resistance. D'Mello et al (1994), for instance, has characterized the $L A G 1$ gene in $\underline{S}$. cerevisiae. It extends life-span, but confers no particular resistance to environmental stress factors at all. The genes RAS1-2 do give resistance to the ionizing effects of UV irradiation (Sun et al, 1992; Kale and Jazwinski, 1997), while SIR-4 creates resistance to heat shock, ethanol and starvation (Kennedy et al, 1995; Martin et al, 1996).

Studies with the nematode Caenorhabditis elegans have also examined the issue of oxidative radicals as the causative agent of aging in two ways. Studies have identified several genes as conferring significant increases to life-span. These include the genes Age-1, daf-2 and spe-26. Age-1 mutants in $\underline{C}$. elegans, live about $65 \%$ longer under normal conditions than wildtype controls and are resistant to the ROS produced by paraquat. Daf-2 and spe-26 increase life-span even more and are all resistant to the effects of paraquat, heat shock and UV (Larsen, 1993; Vanfleteren, 1993). $\underline{\text {. }}$. elegans gives a convincing correlation between long life and the ability to resist free radicals. Long-lived mutants, however, are found to also be resistant to other stresses that suggest the involvement of additional processes in longevity determination.

The third model system for the study of the effects of ROS on aging is Drosophila melanogaster. Far fewer LAGs have been secured in $\underline{D}$. melanogaster than in $\underline{C}$. elegans, but the a priori approach used in tests has both supported the presumptive role for ROS and provoked wider efforts at understanding. In an attempt to enhance the defense against oxidation, Orr and Sohal (1994) constructed a transgenic $\underline{D}$. melanogaster with three additional copies of each gene for production of superoxide-dismutase and catalase. This aimed specifically at enhancing defenses against $\mathrm{H}_{2} \mathrm{O}_{2}$ and its especially destructive derivative, $\bullet \mathrm{OH}$. Prolongation of life only occurred when both superoxide-dismutase and catalase were overexpressed, and presumably provided simultaneous protection against both $\mathrm{H}_{2} \mathrm{O}_{2}$ and $\bullet \mathrm{OH}$. Subsequent experiments failed to find an increase in longevity for either overexpressed Mn-containing superoxide-dismutase or glutathione reductase (Mockett et al, 1999, A,B).

An altogether different attempt has also proven highly successful. Using P-element mutagenesis, Yi-Jyun et al (1998) created a long-lived mutant in $\underline{D}$. melanogaster called Methuselah ( $m t h$ ) that exceeded control populations in maximum life-span by about 35\%. Mth mutants were also highly resistant to paraquat.

In summary then, evidence in support of the "free radical" theory of aging is seen in the fact that (1) the use of oxygen by aerobic organisms releases radicals free in the tissues that render substantial damage to DNA, proteins and lipids. Estimates have placed the proportion of oxidized proteins, in steady state conditions as high as $10 \%$ (Stadtman, 1992). (2) Longer lived organisms resist damage from the herbicide paraquat, which releases some of the same destructive radicals. Thus, resistance to induced damage, of presumably the same type that occurs in natural aging, correlates with long life. And (3), mutants, genetic variants and stocks of longlived organisms created by selection, have also been found in multiple studies to be resistant to paraquat.

In and of itself, this evidence is compelling. Yet, viewed from a larger perspective, it must be realized that though the "free radicals" undoubtedly inflict damage, they constitute but one more environmental challenge that has been overcome in the evolutionary history of any surviving eukaryotic species. The damaging effects of oxygen have been surmounted with sufficient finality that existing flora and fauna don't simply survive, they flourish. Though the antioxidant system clearly fails in existing multicellular forms at later ages, it is one of the oldest of biological defense systems in evolution. It must date in existence from the production of oxygen itself by the first blue-green Cyanobacteria, to between 1.7-2.5 billion years ago. It seems unrealistic to assume that antioxidant defenses have not evolved in that time, to a level of sophistication that completely excludes the corrosive danger from oxygen in the essential reproductive period of life span. This is especially true in view of 
the fact that eukaryotes not only have flourished, but have evolved a number of startling defensive uses for the very substances to which we attribute their eventual demise. Among others, these include the use of superoxide radicals by leukocytes to immobilize and kill invading bacteria and various cleansing and defensive uses of hydrogen peroxide and free radicals by insects. Luckinbill and Foley (2000, in press) describe some of these. The apparent ease with which eukaryotes defend against and control free radicals appears to be totally inconsistent with their eventual destruction by them. How can an effective defense against free radicals be reconciled with our eventual destruction by them in the process of aging? The answer to this may be found by considering the role of oxidants and antioxidants in relation to another basic biological component fundamental to survival.

\section{Empirical Evidence for Other Significant Influences on Aging}

Viewed in its totality, the evidence for oxidation as the ultimate cause of aging is compelling. The corollary of that evidence is that antioxidant enzymes must be the mechanism by which length-of-life is ensured. A substantial amount of other evidence has also been gathered, however, that relates other factors to prolonged life. That evidence, mostly empirical in kind, indicates that the issue of life-span determination may be more complex than simple destruction by oxidative radicals and defense by enzymes. The conclusions of many studies implicate aspects of metabolism other than antioxidants in controlling length of life. One of these involves flight in insects.

The physiology of flight has been thoroughly studied in D. melanogaster. After the first second or two of flight, all energy for subsequent wing strokes comes from glycogen stored in adults. Glycogen is merely long chain glucose, enzymatically polymerized and dehydrated for storage. Glycogen is formed from cytosolic glucose at the very first steps of glycolysis. Stocks of D. melanogaster selected for extended life, fly three or four times longer in experiments than short-lived controls because they have substantially greater quantities of glycogen (Luckinbill et al, 1988; Graves et al 1988; Graves et al 1991). Table 1 indicates some of the other traits found with long life in genetic variants. Most have some known relationship to products of intermediary metabolism, but little direct relationship to oxidants/ antioxidants.

Other comparisons have shown that resistance to starvation and desiccation (not shown in Table 1) also increase in fruitflies with prolonged life. Service et al $(1985,1987)$ found their multiple stocks of $D$. melanogaster were highly durable under those conditions. Although the mechanism of resistance to desiccation is not entirely clear, the ability to resist starvation probably stems from the accumulation of significantly larger deposits of fat (Service et al, 1987) and also glycogen in long-lived adults. Synthesis of greater quan- tities of fat gives a different implication about metabolism in individuals with longer life.

\begin{tabular}{|c|c|c|}
\hline & & \\
\hline $\begin{array}{c}\text { duration of tethered } \\
\text { flight }\end{array}$ & $\begin{array}{c}\text { Synthesis \& storage of } \\
\text { larger quantities of } \\
\text { glycogen }\end{array}$ & $\begin{array}{l}\text { Luckinbill et al (1988) } \\
\text { Graves et al. (1988) } \\
\text { Graves et al (1992) }\end{array}$ \\
\hline resistance to starvation & $\begin{array}{l}\text { synthesis \& storage of } \\
\text { larger quantities of lipid }\end{array}$ & $\begin{array}{c}\text { Service et al (1985) } \\
\text { Service (1987) }\end{array}$ \\
\hline resistance to freezing & $\begin{array}{c}\text { synthesis \& storage of } \\
\text { larger quantities of } \\
\text { glycerol }\end{array}$ & Luckinbill (1988) \\
\hline $\begin{array}{l}\text { maintaining larger } \\
\text { pools of metabolites } \\
\text { from glycolysis } \\
\text { (all of the above) }\end{array}$ & $\begin{array}{l}\text { restriction of carbon } \\
\text { flux through stage I } \\
\text { glycolysis and the } \\
\text { pentose-phosphate } \\
\text { shunt }\end{array}$ & $\begin{array}{c}\text { Riha and Luckinbill } \\
\text { (1996) }\end{array}$ \\
\hline $\begin{array}{l}\text { resistance to heat } \\
\text { shock }\end{array}$ & $\begin{array}{l}\text { production of } h s p 70 \\
\text { and other heat shock } \\
\text { proteins }\end{array}$ & $\begin{array}{l}\text { Lithgow et al (1995) } \\
\text { Khazaeli et al (1997) }\end{array}$ \\
\hline
\end{tabular}

Adults and pupae of long-lived stocks of $\underline{D}$. melanogaster resist the effects of low temperature. Resistance to freezing can be achieved in a variety of different ways. These can range from simply elevating the concentration of glucose or the polyhedric alcohols such as glycerol or sorbitol in cells and blood, to the production of various kinds of thermal hysteresis proteins. In a study by Luckinbill (1998), adult and pupae of D. melanogaster were found to have higher levels of glycerol and resist rapid and slow drop in temperature to $0^{\circ} \mathrm{C}$ or below.

Taken together, the enhanced ability to fly, resistance to starvation, low temperature and perhaps desiccation too, indicate changes in the pattern of metabolism have occured in long-lived individuals. With the exception of desiccation, most of these characters can be associated-with the establishment and maintenance of large metabolite pools of glucose, glycogen, glycerol or other polyols and lipid. It seems at least possible that the dynamics of intermediary metabolism have been altered and somehow may play a role in the establishment of longer life. Specifically, the accumulation and retention of large pools of metabolites at the initial steps of glycolysis suggests a limitation or restriction, if only present temporarily, in the flow of carbon through glycolytic metabolism. This concept is not especially new to aging studies. Sacher (1977) suggested long ago that lowering the "rate of metabolism" would enhance longevity.

The term 'metabolic rate', while seemingly clear in meaning, is difficult to measure with equal precision in all model experimental systems. The term metabolic rate is taken in most instances to refer to the use of $\mathrm{O}_{2}$ during a so-called resting state, that is, the physiological state at which minimal or no physical activity occurs. Under those conditions, the $\mathrm{O}_{2}$ used is taken to indicate the minimum requirement for basic metabolic maintenance or the Basal Metabolic Rate (BMR). In the absence of voluntary muscular activity, the BMR is taken to indicate the rate at which oxidative phosphorylation occurs. 
When used on experimental organisms with clearly identifiable stress behavior, especially in the larger subjects where a single individual is measured, the results unquestionably relate to the actual processes in metabolism. But for invertebrate model experimental organisms with very small body sizes, where measurements of whole populations are made, activity is uncontrolled and for which stress behaviors are unclear and may go undetected, the inferences drawn from $\mathrm{O}_{2}$ use are far less reliable. An alternative method that would be more satisfactory for those subjects, would be to measure the uptake, incorporation and actual flow or flux of carbon through metabolism. To the extent that this method applies to Sacher's inference about metabolic rate and aging, those with reduced carbon flux through metabolism should, have extended life.

On the other hand, not all of the traits of life-span mutants point directly to rate of metabolism. At the other extreme of temperature, adult $\underline{D}$. melanogaster of longlived stocks and mutants of $\underline{\mathrm{C}}$. elegans, have been shown to be resistant to heat shock (Khazaeli et al,1997; Lithgow and Johnson, 1995). While heat and cold shocks are both forms of thermal stress, their mechanisms of resistance are entirely different. The former can be achieved by changing the concentration of simple sugars in common metabolic pathways or their derivatives, but heat shock is resisted by the production and transport of chaperonin proteins such as $h s p 70$. Not only are long-lived stocks resistant to heat shock, but of even greater interest is the conclusion by Khazaeli et al (1997) that adult $\underline{D}$. melanogaster exposed to a sublethal heat shock exhibit an induced increase in longevity. Furthermore, transgenics for which $h s p 70$ genes are overexpressed have an enhanced length of life (Tatar et al, 1997). In neither instance is the life-span extension very substantial, but for both studies the issue is how the hsp70 chaperonin system could accomplish such a feat. This puzzling finding may provide an important vantage for new views of the free radical theory.

The evidence discussed above implicates the involvement of metabolism in determining aging from salient physiological characters that associate with life-span in mutants or stocks. Although much of the initial work exploring these associated traits was done only with $\underline{D}$. melanogaster, studies examining suites of resistance characters are now common with model systems, particularly $\underline{\mathrm{C}}$. elegans. But one other large body of direct experimental evidence relates to the role of metabolism in life-span.

What began in the 1930's as a series of nutritional studies grew later into a more extensive series of examinations before interest finally waned for a time. Then more recent efforts have rededicated themselves to understanding the phenomenon that was described originally. Nutrient or caloric restriction has come to be known as one of the most widely validated, replicated results in aging research. Life is prolonged by simply reducing the intake of food by $20-40 \%$. Levels of intake are meager in that range by comparison with situations of ad libitum intake, but are above the level of malnutrition. No single component of the food is more important than another, but rather it is the total caloric intake produces the observed effect. The extrinsic physiological imposition of this limitation prolongs life in a wide variety of organisms, including many invertebrates, vertebrates, mammals and even higher primates, (Masoro 1991, 1992; Weindruch, 1988). Oddly, however, there are a few instances in which it has not been found. In some cases, this may result from the use of a young organism for which food intake is not yet stabilized (LeBourg and Medioni, 1991). In most cases, however, the effect of caloric restriction is both rapid in effect and independent of age. That is, restriction of calorie intake increases longevity even if it is applied late in life. A suite of other traits also covary with food restriction. Variations in gene expression, immune response, the effect of hormones, behavior and learning, DNA repair, protein synthesis and others have been observed (Sohal and Weindruch, 1996).

\section{A New hypothesis: Antioxidant-Glycation Synergism}

Although alternative hypotheses about the aging process have been formulated, synthesis has been sporadic and limited by fragmentary research. We propose a new synthesis of those hypotheses here, based upon evidence from several areas. First, is that a considerable amount of evidence exists from the study of model experimental aging systems to suggest that metabolic changes accompany increased length of life. For the most part, those studies indicate that where metabolism is restricted sufficiently to cause the accumulation of pools of metabolites, life is long. The exaggerated levels of metabolites are then responsible for the variations in some of the traits that accompany extended life.

How can reducing the flux of carbon through metabolism prolong life? One possibility may be found in the interaction between the production of destructive free radicals, the process of glycation and the opposing action of enzymes in the antioxidant system. Antioxidants scavenge the oxidant radicals given rise to during oxidative phosphorylation. They prevent damage therefore, to cells and tissues, especially the membranes of the mitochondria. Copper-zinc superoxide-dismutase is found in the cytosol while $M n$ superoxide-dismutase is mostly membrane bound in the mitochondrial wall of eukaryotes. Glycation of proteins and lipids occurs spontaneously wherever glucose is found: in blood, tissues, and cytosol. The glyoxidation step in glycation also yields free oxygen radicals that are as destructive in kind as those from oxidative phosphorylation. The process of glycation and free radical production from respiration therefore interact by contributing to the same damaging product. We hypothesize that: (1) the Amadori and AGE products of glycation, produce free radicals in the intermediate steps of the Maillard Reaction, which -contribute to damage and the task of antioxidants (Ortwerth et al, 1998; Elgawish et al, 1996; Carubelli et al, 1995; Gillery et al, 1991). (2) Of undoubtedly greater importance, 
however, is that antioxidant enzymes are stable, longlived, defensive proteins (Fridovich, 1978), and prime targets for glycation themselves (Bourdon, 1999; Staehelin, 1998; Yan and Harding, 1997; Fridovich, 1995). Thus, glycation of antioxidant enzymes should produce the effect seen in Figure 1. That is, glycation produces free radicals and by destroying antioxidants, attacks the very system by which free radicals are removed. A positive synergistic feedback may exist then, between glycation, the level of glucose available to cause glycation, the rate of metabolism, free radical production and suppression of antioxidant level (Figure 1). High levels of blood glucose in ad libitum feeding should foster production of more free radicals and hence reduce available antioxidants. Two other aspects of this interaction are also clear: (1) nutrient or calorie restriction, should lower the concentration of glucose and rate of glycation, lower the yield of radicals and increase levels of antioxidants. (2) The effects of calorie restriction should be rapid, dependent on the rate of increase in restoration of antioxidant enzymes and be reversible, depending on the sustained level of glucose and rate of glycation.

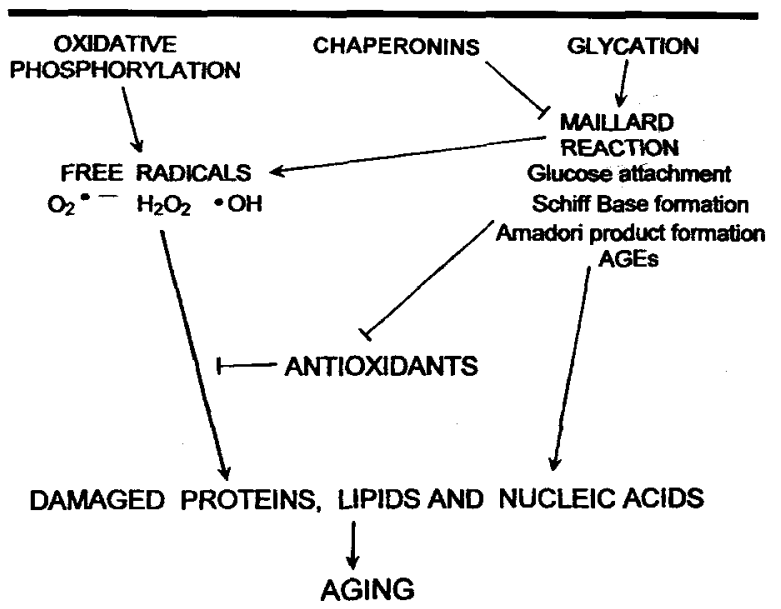

Figure 1. A hypothesized synergism between the glycation process, free oxygen radicals and antioxidants in determining the rate of aging. Advanced glycation end products (AGEs) damage and crosslink proteins, lipids and DNA causing aging. Intermediate steps of the Maillard reaction produce free oxygen radicals that, together with those from oxidative phosphorylation, contribute jointly to molecular damage. Antioxidants protect against damage by scavenging free radicals, but are themselves subject to attack and inactivation by AGE products. A positive feed-back is proposed to exist between glucose level, reduced antioxidant defenses and increased damage by free radicals. Calorie restriction forces glucose levels lower, reducing the interaction with glycation and damage by free radicals. Chaperonins block the formation of Amadori and AGE products. Free radical production from glycation declines and the rate of damage to antioxidant enzymes declines. Antioxidant levels are raised and effectiveness at limiting damage by radicals improves.

The correlation between characters or traits, metabolic rate and life-span of mutants or stocks under selection can be similarly interpreted in terms of this hypothesis. Mutants or long-lived stocks of experimen- tal models should have larger pools of energy-storage metabolites if carbon flow through glycolysis has been reduced. In $\underline{D}$. melanogaster this can be seen in the storage of glucose as lipid and glycogen. The effects on life history and physiological traits of increased levels of these substances has been described, but comparatively few studies have attempted to assess the relative rates of metabolism in long- and short-lived populations.

Gehard and Kauffman (1998), followed by Lee et al (1999), provide such evidence by examining mRNA expression in respectively 588 genes from mouse liver tissue and 6347 genes from mouse skeletal muscle in comparing nutrient restricted mice with controls. Though both studies are generally consistent with the involvement of metabolism with aging, their conclusions are somewhat contrasting (Gehard, 1999). Both are impressive works, but they differ in their interpretation as a consequence of the tissues examined, the elements of the functional groups investigated and even the methods of preparation (Weindruch and Prolla, 1999). A larger problem with these studies is found in the presumption- that the up or down regulation of genes, as indicated by_some rather small changes in mRNA expression, is -reflected in effects on metabolism (and carbon flow).

Only one study has measured the actual carbon flux through glycolysis for comparison of long- and shortlived individuals. Using the stocks of $\underline{D}$. melanogaster selected for prolonged life (Luckinbill et al, 1984), Riha and Luckinbill (1996) measured the flux of carbon through the pentose phosphate shunt in larvae raised under standardized conditions. Several methods have been proposed for the measurement of carbon flux in metabolism, but only that of Cavener and Clegg (1981) is widely accepted as an unambiguous actual measure of flux. Riha and Luckinbill (1996) found that larvae of selected long-lived populations had substantially less uptake, incorporation of labeled glucose into lipid and protein and less measurable flux through the pentose-phosphate shunt. The implication from that result is that selection for long life in D. melanogaster may have copied and enhanced the physiological response occurring under nutrient restriction by promoting expression of the genes control the response.

If the use of glucose in metabolism and the freeradical/antioxidant system relate as we propose, can the bizarre effect of heat shock in extending life also relate to this model? Heat shock proteins belong to a special class of molecule responsible for preserving the stucture and function of other proteins under various stresses. As one of many chaperonin stress proteins, $h s p 70$, for example prevents exposed proteins from denaturing under heat stress. Other chaperonin proteins are known to prevent and/or reverse various steps in the Maillard reaction, that produces the AGE products that crosslink proteins. The stress protein alpha-chrystallin, for example, acts as a molecular chaperon, preventing unfolding and crosslinking of proteins during the Maillard reaction (Derham and harding, 1999; James and Crabbe, 
1998; Hook and harding, 1998). Other chaperonins have been shown to protect enzymes, including antioxidants such as catalase (Hook and Harding, 1996; Ganea and Harding, 1995). In the context of the hypothesis we propose, chaperonins, such as the heat shock proteins, prevent glycation. In doing so, they would reduce the yield of free radicals from the Maillard reaction, and suppress the rate of glycation of antioxidant enzymes. Preventing glycation would act to increase the net levels of antioxidants and thereby also reduce the level of the free radicals. Figure 1 also shows this feedback in the context of the controls proposed.

While the exact role of metabolism in the aging process is far from clear at this time, it seems apparent that it is significant. Evidence is found in: (1) traits or physiological capabilities that come from enlarged pools of particular metabolites that correlate with life-span in experimental model organisms. (2) The vast investigational basis for nutrient restriction indicates simply that reducing carbon flow through metabolism enhances length of life. (3) And finally, two studies of gene expression and one direct comparison of metabolic flux have been performed. Both efforts implicate a significant impact of metabolic rate on life span extension, although the exact elements and functional gene groups remain to be clearly resolved. The third category of evidence shows that the flow of carbon in metabolism exhibits the expected pattern in larvae at least of long-lived stocks.

The experiments discussed are consistent with the "free radical" hypothesis and its derivative, the antioxidant-glycation synergism hypothesis we propose here. Future studies will hopefully isolate the effects of specific LAG genes on both overall metabolism and specific metabolic elements. More precise methods of directly measuring metabolic rate are essential to future progress in this area.

\section{ACKNOWLEDGMENTS}

Drs. Edward Golenberg and D. C. Freeman were kind enough to read earlier drafts of this work.

\section{REFERENCES}

1. Ames, BN, Shigenaga, MK and Hagen, TM: Oxidants, antioxidants and the degenerative diseases of aging. Proc. Nat. Acad. Sci., 90:7915-7922, 1993.

2. Agarwal, S and Sohal, RS: DNA oxidative damage and life expectancy in houseflies. Proc. Nat. Acad. Sci., 91:12332-12335, 1994.

3. Bourdon, E, Loreau, N and Blache, D: Glucose and free radicals impair the antioxidant properties of serum albumin. Faseb Jour. 13:233-244, 1999.

4. Carubelli, R, Schneider, JE, Pye, QN and Floyd, RA: Cytotoxic effects of autoxidative glycation. Free Rad. Biol. Med. 18:265-269, 1995
5. Cavener, D and Clegg, M: Evidence for biochemical and pysiological differences between genotypes in Drosophila melanogaster. Proc. Nat. Acad. Sci. 78:4444-4447, 1981.

6. Cerami, A: A hypothesis: Glucose as mediator of aging. J. Am. Geriatr. Soc., 33:626-634, 1985.

7. D'Mello, NP, Childress, AM, Franklin DS, Kale, SP, Pinswadi, C and Jazwinski SM: Cloning and characterization of LAG1, a longevity assurance gene in yeast. Jour. Biol. Chem., 269:15451-15451, 1994.

8. Elgawish, A, Glomb, M, Friedlander, M and Monnier VM: Involvement of hydrogen peroxide in collagen cross-linking by high glucose in vitro and in vivo. Jour. Biol. Chem. 271:12964-12971, 1996.

9. Fridovich, I:The biology of oxygen radicals. Science 201:875-880, 1978

10. Fridovich, I: Superoxide radical and superoxide dismutases. Ann. Rev. Biochem. 64:97-112, 1995.

11. Ganea, E and Harding, JJ: Molecular chaperones protect against glycation-induced inactivation of glucose-6-phosohate-dehydrogenase. Eur J Biochem. 231:181-185, 1995.

12. Gehard, GS: Cutting back the calories. Science 286:1679, 1999.

13. Gehard, GS and Kauffman, E: High throughput gene expression analysis in mice subject to caloric restriction. Gerontol. Abstr. 38:16-16, 1998.

14. Gillery, P, Monboisse, JC, Maquart, FX and Borel, JP: Aging mechanisms of proteins. Diabetes and metabolism. 17:1-15, 1991.

15. Gorham, BK and Harding, JJ: Alpha-chrystallin as a molecular chaperone. Prog. Retin. ye Res. 18:463509, 1999.

16. Graves, JL, Luckinbill, LS and Nichols, A: The effect of selection for life-span in Drosophila: flight duration and measurment of wing beat frequency. J. Insect Physiol., 34:1021-1026, 1988.

17. Graves, JL, Toolson, EC, Jeong, C, Vu, LN and Rose, MR: Desiccation, flight, glycogen andf podtponed senescence in Drosophila melanogaster. Physiol. Zool., 65:268-286, 1992.

18. Harman, D: Aging- a theory based on free radical and radiation chemistry. Jour. Gerontol. 11:298300, 1956.

19. Hook, DWA and Harding, JJ: Protection of enzymes by alpha-chrystallin acting as a molecular chaperone. Int. J. Biol. Macromol. 22:295-306, 1998 (A).

20. Hook, DWA and Harding JJ: Alpha-chrystallin acting as a molecular chaperone protects catalase against steroid-induced inactivation. Febs Lett. 382:281-284, 1996 (B). 
21. James, $\mathrm{M}$ and Crabbe, $\mathrm{C}$ : Cataract as a conformational disease: The Maillard reaction, alphachrystallin and chemotherapy. Cell Molec. Bio. 44:1047-1050, 1998.

22. Jazwinski, JM, in Handbook of the biology of aging, edited by Rowe, JW and Schneider, EL, New York, Academic Press, pp. 41-54, 1990.

23. Jazwinski, SM: Longevity, genes and aging. Science, 273:54-59, 1996

24. Kale, SP and Jazwinski, SM: Differential response to UV stress and DNA damage during the yeast replicative life sapn. Dev. Gen., 18:154-160, 1996.

25. Khazaeli, A, Tatar, M, Pletcher, S and Curtsinger, J: Efects of heatshock on life history in Drosophila melanogaster : Age-specific mortality, reproduction and thermotolerance. J Gerontol., 52A:B48-B52, 1997.

26. Kennedy, BK, Austriaco, NR, Jr. Zhanp, J and Guarente, L: Mutation in the silencing gene of SIR4 can delay aging in S. cerevisiae. Cell 80:485-486, 1995.

27. Lee, CL, Klopp, RG, Weindruch, R and Prolla, T: Gene expression profile and its retardation by caloric restriction. Science 285:1390-1393, 1999.

28. Lithgow, GJ, White, TM, Melov, S and Johnson TE: Thermotolerance and extended life-span conferred by single-gene mutations and induced by thermal stress. Proc. Nat. Acad. Sci., 92:7540-7544, 1995.

29. Larsen, PL: Aging and resistance to oxidative damage in Caenorhabditis elegans. Proc. Nat. Acad. Sci., 90:8905-8909, 1993.

30. LeBourg, E, and Medioni, J: Food restriction and longevity in Drosophila melanogaster. Age and Nutr. 2:90-93, 1991.

31. Lin, SJ and Culotta, VC:The ATX1 gene of Saccharomyces cerevisiae encodes a small metal homeostasis factor that protects cells against reactive oxygen toxicity. Proc. Nat. Acad. Sci., 92:37843788, 1995.

32. Lin, SJ and Culotta, VC: Suppression of oxidative damage by Saccharomyces cerevisiae ATX2: Which encodes a manganese-trafficking protein that localizes to Golgi-like vesicles. Mol. Cell Biol., 16:63036312, 1996.

33. Luckinbill, L and Foley, P: An analysis of experimental and empirical approaches in the study of aging. Biogerontol., 1: (In press), 1999.

34. Luckinbill, LS: Selection for longevity confers low temperature resistance in Drosophila melanogaster. J. Gerontol., 53:B147-B153, 1998.

35. Luckinbill, LS, Arking, RA, Clare, MJ, Cirocco, WC and Buck, SA: Selection for delayed senescence in Drosophila melanogaster. Evol. 38:996-1003, 1984
36. Luckinbill, LS, Graves, JL, Tomkiu, A and Srowirka, $O$ : A qualitative analysis of some correlates of longevity in Drosophila melanogaster. Evol. Ecol., 2:85-94, 1988.

37. Martin, GM, Austad SN and Johnson, TE: Genetic analysis of ageing: Role of oxidative damage and environmental stresses. Nat. Gen. 13:25-34, 1996.

38. Masoro, EJ, Shimokawa, I and Yu, BP: Retardation of the aging process in rats by food restriction. Ann. N.Y. Acad. Sci., 621:337-352, 1991.

39. Masoro, EJ: Aging and proliferative homeostasis: Modulation by food restriction in rodents. Lab Anim. Med.,42:132-137, 1992.

40. Matsuo, M, Gomi, F and Dooley, MM: Age related alterations in antioxidant capacity and lipid peroxidation in brain, liver and lung homogenates of normal and vitamin-E-deficient rats. Mech. Aging Dev., 64:273-292, 1992.

41. Metchnikoff, E: The prolongation of life: Optimistic studies. New York, GP, Putnam's Sons, 1908.

42. Mockett, RJ, Orr, WC, Rahmandar, JJ, Benes, JJ, Radyuk, SN, Klichko, VI and Sohal, RS: Overexpression of Mn-containing superoxide-dismutase in transgenic Drosophila melanogaster. Arch. Biochem. Biophys. 371:260-269, 1999 (A)

43. Mockett, RJ, Sohal, RS and Orr, WC: Overexpression of glutathione reductase extends survival in transgenic Drosophila melanogaster under hyperoxia but not normoxia. Faseb Jour. 13:17331742, 1999 (B).

44. Molisch, H: The Longevity of Plants. New York, Botanical Garden, 1938.

45. Orr, W and Sohal, RS: Extension of lifespan by the overexpression of superoxide-dismutase and catalase. Science 163:1128-1130, 1994.

46. Osiewacz, HD. Aging and genetic instabilities, in Molecular aspects of aging, edited by Esser, $\mathrm{K}$ and Martin, GM, Chichester, John Wiley and Sons, 1995, pp 28-44.

47. Ortwerth, BJ, James, H, Simpson, G and Linetsky $M$ : The generation of superoxide anions in glycation reactions with sugars, osones and 3-deoxysones. Biochem. Biophys. Comm. 145:161-165, 1998.

48. Riha, V and Luckinbill, LS: Selection for longevity favors stringent metabolic control in Drosophila melanogaster. Jour. Gerontol.51:B284-B294, 1996.

49. Sacher, GA: in Handbook of the biology of aging, edited by Finch, CE and Hayflick, L, New York, Van Nostrand, Reinhold, 1977. pp 581-538.

50. Sagai, $M$ and lchanose, $T$ : Age related changes in lipid peroxidiation as measured by ethane, ethylene, butane and pentane in respired gasses of rats. Life Sci., 27:731-738, 1980. 
51. Service, PM: Physiological mechanisms of increased stress resistance in Drosophila melanogaster selected for postponed senescence. Physiol. Zool., 60:321-326, 1987.

52. Service, PM, Hutchinson, EW, MacKinley, MD and Rose MR: Resistance to environmental stress in Drosophila melanogaster selected for postponed senescence. Physiol. Zool., 58:380-389, 1985.

53. Shieganaga, MK, Hagen, TM, and Aames, BN: Oxidative damage and mitochondrial decay in aging. Proc. Nat. Acad. Sci., 91:10771-10778, 1994.

54. Simonian NA and Coyle, JT: Oxidative stress in neurodegenerative dieases. Ann. Rev. Pharmacol. and Toxicol., 36:83-106, 1996.

55. Sohal, RS and Weindruch, R: Oxidative stress, caloric restriction and aging. Science 273:59-63, 1996.

56. Sun, J, Kale, SP, Childress, AM, Pinswasdi, C, and Jazwinski, SM: Divergent roles of RAS1 and RAS2 in yeast longevity. J. Biol. Chem., 269:18638-18645, 1994.

57. Staehelin, HB: Free radicals and glyoxidative stress in ageing and age-rrelated diseases. Austral. Jour. Ageing 17:85-87 Suppl. S, 1998.

58. Stadtman, ER: Protein oxidation and aging. Science :257:1220-1224, 1992.

59. Tatar, M, Khazaeli, A and Curtzinger, J: Chaperoning extended life. Nature 390:30, 1997.

60. Vanfleteren, JR: Oxidative stress and aging in Caenorhabditis elegans. Biochem. J., 292:605$608,1993$.

61. Wallace, DC. Mitochondrial DNA mutations and bioenergetic 11. defects in aging and degenerative diseases, in Molecular and genetic basis of neurologic disease, edited by Rosenburg, DN, Pruisner, SB, DiMauro, S, and Barchi, RL, Bostson, Butterworth Hineman, 1996.

62. Weindruch, R, and Prolla, TA. Response. Science 286: 1679-1670, 1999.

63. Weindruch, $R$, and Walford, RL. The retardation of aging and disease by dietary restriction. Springfield Illinois, Thomas, 1988.

64. Yan, $\mathrm{H}$, Harding, JJ: Glycation induced inactivation and loss of antigenecity of catalase and superoxide-dismutase. Biochem. J. 328:599-605, 1997.

65. Yim, MB, Kang, JH, Yim, HS, Kwak, HS, Chock, PB and Stadtman, ER: A gain-of-function of an amyotrrophic lateral sclerosis-associated $\mathrm{Cu}, \mathrm{Zn}$ superoxide-dismutase mutant: An enhancement of the free radical formation due to a decrease in $\mathrm{K}_{\mathrm{m}}$ for hydrogen peroxide: Proc. Nat. Acad. Sci., 93:57095714, 1996.
66. Lin, YJ, Seroude, L and Benzer S: Extended lifespan and stress resistance in the Drosophila mutant methusaelah. Science 181:943-946, 1998. 